The second diagram refers to a similar set of 4380 thermometric observations ( $\mathrm{I}$ ) of the 1 aximum, (2) of the minimum temperature on successive days ${ }^{1}$ from January 1,1873 .

In regard to the first diagram the asymmetry is obvious. I have tested the conclusion in the usual way. For instance, the total of 4857 observations was composed of seven batches of a little less than two years each. Precisely the same asymmetry, in varying degrees, is displayed by each of these batches. The asymmetry is of course obvious to the eye in the diagram, but various numerical tests may be proposed. For instance, we may compare ( $\mathbf{I}$ ) the position of the mean value (in this case $29^{\circ} .9 \mathrm{I}$ ) between the extreme values, (2) the relative positions of the maximum ordinate and the mean ordinate, $(3)$ the comparative magnitudes of the "mean errors" to the right and the left of the mean ordinate. They all yield a result in the same direction.

I should be very glad if any of your readers could confirm (or correct) these results by those of more extended observations, or by results taken from other districts. That something of this kind should be displayed where, as here, we are dealing with a one-ended phenomenon-i.e. with one in which unlimited variation was conceivable in one direction but not in the otherseems to me in itself reasonable. But I was certainly surprised to find it so marked, considering how small is the fluctuation in relation to the actual magnitude of the variable phenomenon.

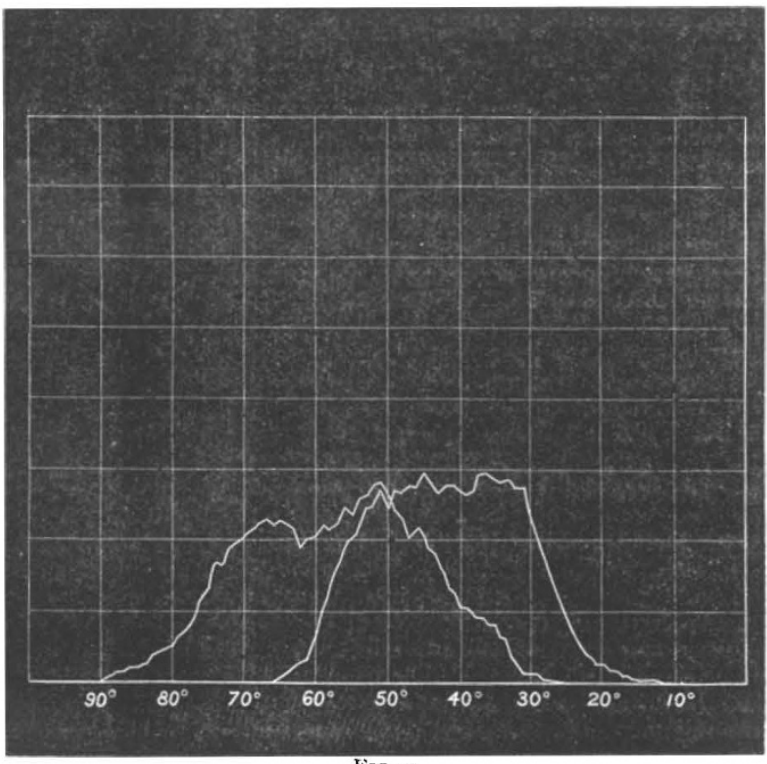

FIG. 2 .

It seems to suggest that the common theoretic assumption of a sort of fixed mean or type which is swayed about by a large number of equal aud opposite independent disturbing causes, does not hold good in this case.

As regards the second diagram, the two curves are (especially that of the minima) tolerably symmetrical, but they depart widely from anything approaching to Quetelet's supposed fixed type.

Anyone looking at the curve of maxima would say at once that it mingled the results of two distinct means (in Quetelet's phrase), as if we were to group together the observed statures of a great many Scotchmen and Frenchmen. That we are mingling results of distinct means seems true enough, but not of two such, and I cannot account for the two peaks in the curve. What I should have expected would have been something of this kind: Each day has its own appropriate mean maximum (subject to the usual fuctuation), and these mean maxima are themselves grouped about their mean, hence the true mean of all ought to be decidedly the commonest result, i.e. the curve should have a single vertex.

The facts are quite otherwise. The depression towards the

${ }^{2}$ In this case, as the lengths of the successive ordinates from the original data were very irregular. I have smoothed the curve out by taking the mean of three successive heights. For instance, to take the actual figures, the number of occasions on which the maxima were $58^{\circ}, 59^{\circ}$, and $60^{\circ}$, were respectively 108,99 , and 124 ; I have assigned the number 110 to $59^{\circ}$, and so on. centre is far too deep to be accidental, and the final mean (i.e. about $57^{\circ}$ ) is very far from being the commonest value.

Somewhat similar remarks may be made about the curve of minima. There is some evidence (though not conclusive) of a depression towards the centre in this case also, and the curve is very fairly symmetrical. But the true mean of all the minima cannot claim any numerical preponderance over any other value between $32^{\circ}$ and $52^{\circ}$.

I am far too deeply conscious of the numerous pitfalls which lurk about the statistician's path to offer these results with any great confidence. But considering how large is the number of observations included, it certainly seems to me that they call for some explanation. There may of course be some blunder in the calculations, but $\mathrm{I}$ have done my best to guard against this. What I trust is that these results may be the means of calling forth some discussion by practised experts in this branch of statistical inquiry, which may serve to confirm or correct my results, and in the former case to offer some explanation of the causes of the phenomena. Very likely this practical inquiry has been already undertaken elsewhere, but the statistics of meteorology are so vastly extensive that it is impossible for any but a professional student of the subject to be acquainted with what goes on in it.

Cambridge.

J. VENN.

\section{The Sense of Smell in Dogs.}

Will Mr. Russell (whose letter in Nature of August 4 I have just read) be so good as to make another experiment with his pug bitch? He says that she had been "taught to hunt" for biscuit ; probably she was also enjoined to "find it," or something similar, when she came into the room. Can he manage to try her powers without awakening her expectation?

$I$ ask it because it seems to me that in this case (and many others) we have something different to observe than mere quickness or keenness of sense, and something well worthy of observation; namely, exclusive direction of the attention of a senseif I may so term it.

We may note this mysterious power in ourselves to a certain extent. In the case of a dog or bird, or any other in which there is little brain work going on to cause distraction, it may be much greater, and account for many wonderful things. It may be said that this is trying to explain the unknown by the even less known; nevertheless, by gathering together many and varied instances of the action of any power some light must be thrown upon it. The mesmerizer seems to deal with this one when he closes all avenues to the senses of his subject except the one he wishes to keep open.

The sense of hearing in some birds seems as wonderful and discriminating as that of smell in dogs. I have watched with astonishment a thrush listening for worms-as their manner isand very evidently hearing them too, within two yards of a noisy lawn-mower on the other side of a small hedge of roses. Probably the worms came nearer to the surface in consequence of the vibration caused by the machine--they are said to do so -but that the thrush heard and did not see them was evident. Robins appear to be able to distinguish the voices of their own offspring and parents from a number of others, and at a great distance. I say appear, for in such a case one cannot be quite sure, still less can one give all the small details of long-continued observation that make up the evidence in favour of it.

All these cases have a common and mysterious element. It is as if a window were opened in one direction and all others closed; or a chord set vibrating that answers, as a struck glass answers, only to one note; or as if all the available energy were directed along one narrow path. At any rate there is something more than mere keenness of sense.

Sidmouth.

\section{Electricity of Contact of Gases with Liquids.}

Will you allow me to ask Mr. Enright (NATURE, p. 365) how he proved that the "charge of the escaping hydrogen was positive" or negative, as the case may be? That the escaping spray was electrified by friction, after the manner of the steam spray in Armstrong's old hydro-electric machine, is a natural explanation of these capricious effects; but that gas should be thus electrified, and that this electrification should have any relation whatever to the subject of "atomic charge," are propositions which strike one as improbable.

Oliver J. LODGr. 